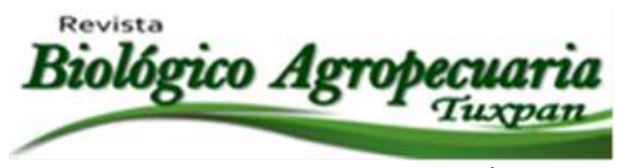

\title{
La depresión infantil y su vinculación con la falta de valores en la familia
}

Child depression and its link with the lack of values in the family

Araiza Santillán Marisa Aideé ${ }^{1 凶}$, Palafox Bolívar Marisol Priscila ${ }^{1}$, Domínguez Arrieta Eduardo $^{1}$, Sandoval Lujan Graciela del Carmen ${ }^{1}$ y Torres López Octavio ${ }^{1}$

Universidad Autónoma de Chihuahua, Facultad de Contaduría y Administración. Calle $6^{\text {a y ave }}$ 21, pte sin número. Ciudad Delicias Chihuahua, Chihuahua.

${ }^{\circledR}$ Autor para correspondencia: maraiza@uach.mx

Recibido: $15 / 04 / 2019$

Aceptado: 15/05/2019

\section{RESUMEN}

El proceso de crecimiento del ser humano es complejo y variable, a lo largo de su vida se enfrenta a diferentes escenarios y aspectos que la sociedad impone como parte del desarrollo "normal" de todo individuo que culminan en la formación de una familia y la procreación de nuevos seres humanos para completar el entornos familiar idealizado.

Sin embargo, la sociedad ha evolucionado y se ha vuelto más compleja por toda la información, el desarrollo y la tecnología, que a la vez que genera progreso. Lo anterior crea confusión entre los miembros de la familia al desviar lo que por mucho tiempo se consideró prioridad para los padres como la unión, el desarrollo y fortalecimiento de valores familiares.

Con la evolución de la sociedad, las prioridades se centran en el crecimiento profesional de los padres sin prever las necesidades básicas de sus hijos, en la mayoría de los casos, en que la participación económica de la madre se ha hecho cada vez más importante, y a la vez, no se ha planeado la evolución entre los tres conceptos básicos: Familia-Valores y Estabilidad Moral.

Cuando en una familia no hay participación activa de los padres en la educación de los hijos, se crea un vacío de soledad y de sentido de ausencia, lo cual ha provocando un incremento del síndrome llamado "Depresión infantil", que va en ascenso desde hace 10 años a la fecha. Los síntomas que se manifiestan son la actitud de ausencia, retraimiento e irritabilidad en los niños, lo cual marca su desarrollo y el de los padres. El alto índice de crecimiento de este síndrome está creando un futuro incierto para: padres e hijos al no saber el tipo de traumas, conflictos emocionales y/o los trastornos de identidad que desarrollaran los hijos y a los que se enfrentarán los padres al mismo tiempo; la sociedad al ser incierto el tipo de familias que se establecerán en el futuro; y los individuos afectados psicológicamente que no puedan expresar o recibir muestras de afecto o que al no haber recibido afecto, no puedan sentirlo y desarrollarse como un individuo dentro de la sociedad.

Es responsabilidad de nosotros como padres y como miembros de una sociedad el ayudar a que este síndrome depresivo disminuya y a que podamos formar jóvenes libres en de mente y en de espíritu, jóvenes que puedan darnos un México mejor y que puedan librarse de trastornos emocionales que los lleven formar familias disfuncionales, y seguir con este síndrome en crecimiento. 
El presente trabajo identifico las principales características que refleja un niño con este síndrome, así como, la manera en que se puede atacar estimulando el crecimiento familiar y visualizar el impacto que se genera con técnicas básicas y elementales.

Palabras clave: Depresión Infantil (DI), Valores y Desarrollo Familiar.

\begin{abstract}
The process of growth of the human being is complex and variable, throughout his life he faces different scenarios and aspects that society imposes as part of the "normal" development of every individual that culminates in the formation of a family and procreation of new human beings to complete the idealized family environment.

However, society has evolved and has become more complex for all information, development and technology, while generating progress. This creates confusion among family members by diverting what was long considered a priority for parents such as union, development and strengthening family values. With the evolution of society, the priorities are focused on the professional growth of the parents without anticipating the basic needs of their children, in most cases, in which the mother's economic participation has become increasingly important, and at the same time, the evolution between the three basic concepts has not been planned: Family-Values and Moral Stability.

When there is no active participation of parents in the education of children in a family, a vacuum of loneliness and sense of absence is created, which has led to an increase in the syndrome called "Child Depression", which has been on the rise for a long time. 10 years to date. The symptoms that manifest are the attitude of absence, withdrawal and irritability in children, which marks their development and that of the parents. The high growth rate of this syndrome is creating an uncertain future for: parents and children not knowing the type of trauma, emotional conflicts and / or identity disorders that children will develop and which parents will face at the same time; society being uncertain the type of families that will be established in the future; and psychologically affected individuals who cannot express or receive signs of affection or who, having not received affection, cannot feel it and develop as an individual within society.

It is our responsibility as parents and as members of a society to help reduce this depressive syndrome and to be able to train young people free in mind and spirit, young people who can give us a better Mexico and who can get rid of emotional disorders that take form dysfunctional families, and continue with this growing syndrome.

The present work identified the main characteristics that a child with this syndrome reflects, as well as the way in which it can be attacked by stimulating family growth and visualizing the impact that is generated with basic and elementary techniques.
\end{abstract}

Keywords: Child Depression (ID), Values and Family Development. 


\section{INTRODUCCIÓN}

El desarrollo del entorno familiar en la vida cotidiana ha sufrido muchos cambios, entre los que se encuentran el desarrollo físico y mental de los hijos en las familias en México, en particular, en los últimos años la ruptura entre la relación padres e hijos, la falta de valores en el medio social, la comunicación del exterior a la familia y la lucha por sostener los valores, han sido un detonante para el incremento de Síndrome Depresivo en la población infantil. Esta situación llevó a realizar una investigación de los fenómenos y situaciones que causan el síndrome, así como los efectos que se presentan en la comunidad debido a dicha enfermedad. Detener la Depresión Infantil (DI) es prioridad porque los niños de hoy serán los adultos del futuro y estarán encargados de sostener la sociedad por lo que es imperante que los niños se desarrollen en un entorno sano y seguro que les brinde energía física y emocional para lograr sus metas, tener sueños e ilusiones. Si el síndrome depresivo se presenta en los infantes y no es atendido, el síndrome los acompañará a través de la adolescencia y la adultez trayendo como consecuencia grupos sociales que crecerán de manera disfuncional dentro del eje familiar, dentro de estos ejes, el más importante es la familia, la cual constituye la base del crecimiento de nuestra sociedad, por lo que es importante dedicarle tiempo al estudio de las causas y efectos de los valores internos dentro de la misma, así como el efecto directo que se sufre en relación a la atención de los niños y adolecentes dentro del seno familiar.

El incremento importante se ha visto dentro del periodo de 10 años atrás, lo que ha preocupado a familias, grupos educativos $\mathrm{y}$ psicólogos que han visto cómo las necesidades de los niños han ido evolucionando, pero a la vez las necesidades de los padres también, por lo que es necesario que tanto sociedad, familia y grupos religiosos, aportemos un poco de lo mucho que se requiere para que esta sociedad cambie y evolucione, a tal grado que se pueda lograr un ambiente sano, y lleno de familias en pro del desarrollo humano completo y plenamente satisfactorio.

\section{MATERIALES Y MÉTODOS}

Cambios en las familias

En un periodo de 10 años aproximadamente, se ha visualizar un gran cambio que ha surgido en las familias, en especial de las familias en México, y aquí es donde se ve que los valores en general han ido en declive.

Algunos cambios que se han podido visualizar en la familia según (Merino, 2007), son los siguientes:

La aceleración de la pérdida de valores religiosos, La afirmación de Merino está considerada la más importante, ya que al no tener en que creer, no crecemos, y mientras existan sueños y fe, habrá esperanza de cambio, los sueños nos permiten seguir viviendo $\mathrm{y}$ confiando.

La pérdida de fronteras de los nuevos medios de comunicación social (redes sociales). Como ya se mencionó, Merino acertadamente acentuó la apertura a la comunicación como un arma de dos filos, la cual ha desembocado en una puerta a la ausencia y alejamiento del niño/joven con su familia, llevándolo a un individualismo impresionante, que genera soledad y tristeza, envuelta en una capa de diversión.

La generación de una sociedad más plural y multicultural. El autor señaló de manera clara que muchas veces al querer alcanzar el desarrollo educativo, cultural y global a que lleva los procesos de cambio, y el querer adoptar costumbres e ideas diferentes a nuestra cultura y 
tradiciones, que muchas veces van en contra de la cultura familiar.

La incorporación de la mujer en su nuevo rol dejando al cuidado directo del hogar para incorporarse a la fuerza laboral y del conocimiento. En concordancia con el autor en relación a este punto, es de suma importancia hacer mención que la mujer ha tenido que evolucionar, pero que esto no lleva consigo el abandono de los valores individuales de su familia, o bien la enseñanza de los mismos, a sus hijos, sino al contrario, es el crecer y enseñar a sus hijos a desarrollarse y a reafirmar los valores básicos, para perder la base del entorno familiar. El crecimiento de las ciudades y la configuración de nuevos valores, así como la problemática de la buena comunicación. El crecimiento globalizado de la sociedad, lleva a la pérdida de valores y de las bases solidas de la familia, pero este debería ser un punto de desarrollo positivo, ya que, el crecimiento en si lleva al progreso, pero él no llevarlo a cabo de manera adecuada, lleva a que este punto se convierta en un factor negativo para el buen desarrollo familiar.

De esta forma entonces se puede ver, que todos estos factores han ido marcando considerablemente los cambios en las relaciones familiares, pero ¿porque los niños lo han resentido tanto al grado de llegar padecer de Depresión Infantil (DI)?, para empezar se analizará cual es el concepto de "Depresión infantil", y así se podrá entender ampliamente él porqué es tan impactante este fenómeno dentro de nuestra sociedad.

Depresión infantil

La depresión infantil(DI) según (Rodriguez Avila \& Ortiz Ramirez, 2008) nos dicen que es "Un desorden caracterizado por una alteración en el estado de ánimo, acompañada de cambios en el comportamiento a nivel escolar, familiar y social" , considerándose este concepto por los autores como una enfermedad típica de la gente adulta en los años anteriores, pero en la actualidad se le ha podido dar la importancia que tiene, pudiendo analizar que una de las principales causas de la misma es la falta de afecto, o bien la carencia del mismo. Y viéndolo desde el punto de vista general, volvemos a lo más prioritario...la familia, ya que si desmenuzamos en concepto, veremos que ciertamente como indicó el autor ese desánimo y falta de interés en sus actividades cotidianas, generalmente las confundimos con flojera o falta de responsabilidad, creando en el niño/joven un sentido de culpa y de menosprecio, acompañado de el habitual castigo o represión que tendrán por parte de sus padres, en caso de que lo detecten, si no, pues simplemente pasará por alto, y crecerá sin la visualización correcta de sus actos. (Rodriguez Avila \& Ortiz Ramirez, 2008) Mencionan también que la DI puede definirse como "una situación afectiva de tristeza mayor que ocurre en un niño". Y con esto se corrobora el concepto anterior y se verifica que los conceptos de depresión se relacionan de manera amplia con "tristeza" y "soledad". Por lo anterior nacen las siguientes preguntas:

¿Qué estamos haciendo, que tipo de niños van a desarrollarse para el nuestro futuro?

¿Qué provoca al niño/joven a sentir depresión?

Muchas veces nos podemos hacer estas preguntas, y pudiéramos centrarla en que tal vez ellos no encuentran su lugar en la sociedad, en el vínculo familiar, escolar o que no están maduros emocionalmente para enfrentar la vida como nosotros quisiéramos, pero no es así, los niños, en su mayoría están ávidos de aceptación, de sentirse útiles en sus hogares, de no ser puntos de comparación con sus hermanos mayores, o menores en su caso, o bien con algún familiar 
cercano ellos, tal vez la falta de acercamiento de los padres y la falta de comunicación entre ellos, ha generado que ellos muestren una inestabilidad emocional, ahora denominada "Depresión infantil", en (Boyd, 2003) podemos captar la idea de depresión infantil en una redacción que se les pidió a niños de educación media, en las que les piden describan como seria su propio epitafio donde una joven de características alegres, escribió lo siguiente:

"Había una vez una muchacha, que se llamaba Sara. Donde por fuera parecía perfecta, pero por dentro tenía muchos problemas, debido a que era la mayor de cuatro hermanas, era mucha la presión que ponían en ella. Ya no podía soportar más la vida, sus notas bajaban cada vez más y sus principios morales no eran los correctos. Sara salió con sus supuestos "amigos", y nunca regresó al hogar, su cuerpo sin vida fue encontrado flotando en el río el 2 de Julio de 1992".

Esta redacción elaborada por una joven de educación media (secundaria), denota el vacio y la soledad existente en su vida, tal vez no por la falta de un vinculo familiar, porque como podemos ver ella tenía 3 hermanas más, sino por la falta de aceptación y la existencia de comparación entre ella y "alguien más", que tal pudieran ser sus hermanas, sus familiares o bien hasta sus mismos padres, y esto genera un proceso de depresión, que puede llegar a ser caótico y fatal en el niño y/o joven.

Cuando se presenta este fenómeno, entonces quiere decir que ya se llega a un extremo en la relación familiar, la aceptación entre ellos y la comunicación entre padres e hijos y lo más importante, la función de la mamá en cuanto a las reacciones de sus hijos, porque es triste y desmotivante el hecho de ver que ni siquiera los padres se dan cuenta del momento preciso en el que se presenta de DI, sino al contrario, se pasa por alto, y se puede confundir con parte de su desarrollo, en lugar de ver la profundidad de sus sentimientos, los padres deben conocer a sus hijos, y poder identificar sus debilidades y sentimientos para poder ayudarlos y sacarlos del fondo obscuro en el que muchas veces están.

Actuación de la mujer en el proceso de desarrollo de la familia. En relación a este punto, se puede acentuar la participación de la madre en este proceso, (Voltas, 2008) dice que "debemos dar gracias directamente a las mujeres, a cada mujer, por lo que representan en la vida: gracias, madres, que sois el seno y primer recinto del ser humano en una experiencia única que os convierte en guía y punto de referencia en la vida de vuestros hijos", aquí se denota la importancia que Voltas le da a la gran responsabilidad que tiene la mujer dentro del vinculo familiar, pero cabe mencionar que en esta época, donde la mujer, como anteriormente de mencionó, busca ocupar un lugar en la sociedad, en la vida laboral y profesional, y con el afán de crecer y dar mayor seguridad económica a sus hijos, se ha desbordado en crecer y desarrollarse ella misma y ha dejado lo prioritario por lo importante, que es la relación y la atención que debe tener con sus hijos.

En las siguientes gráficas del Instituto Nacional de Estadísticas (INE, 2007), así como del Hospital General de México (HGM, 2011) se muestra el incremento considerable que se ha presentado en las mujeres que trabajan y el alto índice de niños con DI que a la par con lo anterior, se ha ido desarrollando, aun y cuando en los últimos años disminuyó, ya sea por falta de atención (por lo que no queda registrado) como por disminución de la misma.

La evolución de la mujer marca de gran manera el crecimiento humano, pero si lo reforzara con la evolución familiar, sería un gran acierto su desarrollo, desgraciadamente, en las 
siguientes gráficas se verán los dos conceptos, que han crecido a la par, es decir que se muestra la relación directamente proporcional entre el desarrollo profesional de la mujer y el aumento de la depresión infantil. Si logramos que en la proporción que la mujer se desarrolla, disminuye en índice de niños /jóvenes con depresión, entonces, el trabajo de los proyectos de familia, resultaran exitosos.

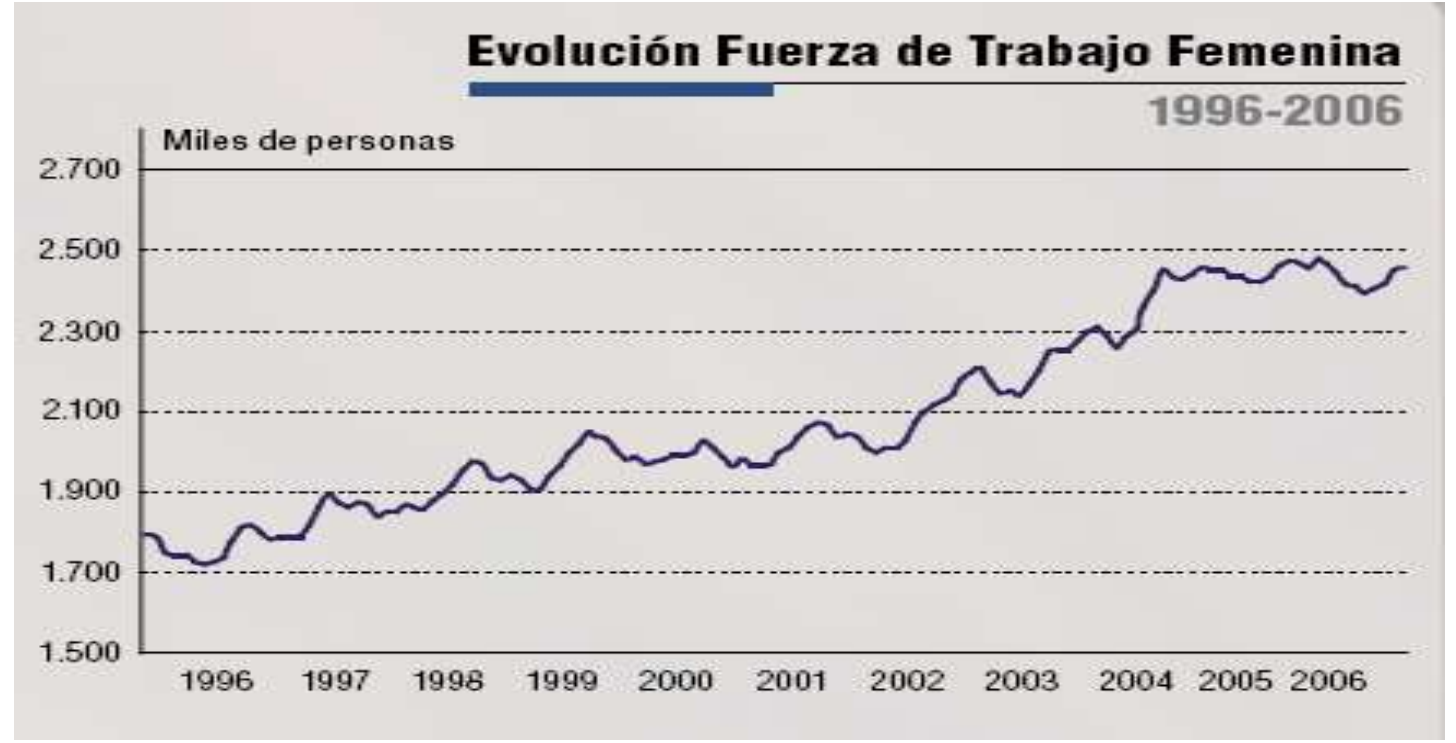

Figura 1. Evolución de fuerza de trabajo femenina. Fuente: Instituto Nacional de estadísticas (INE) del 9 de marzo del 2007.

En esta gráfica, podemos identificar qué gran avance ha sido la evolución de la mujer dentro del área laboral y lo impactante del crecimiento grafico de la mujer dentro del área laboral, que no es más que las ganas de desarrollarse y evolucionar.

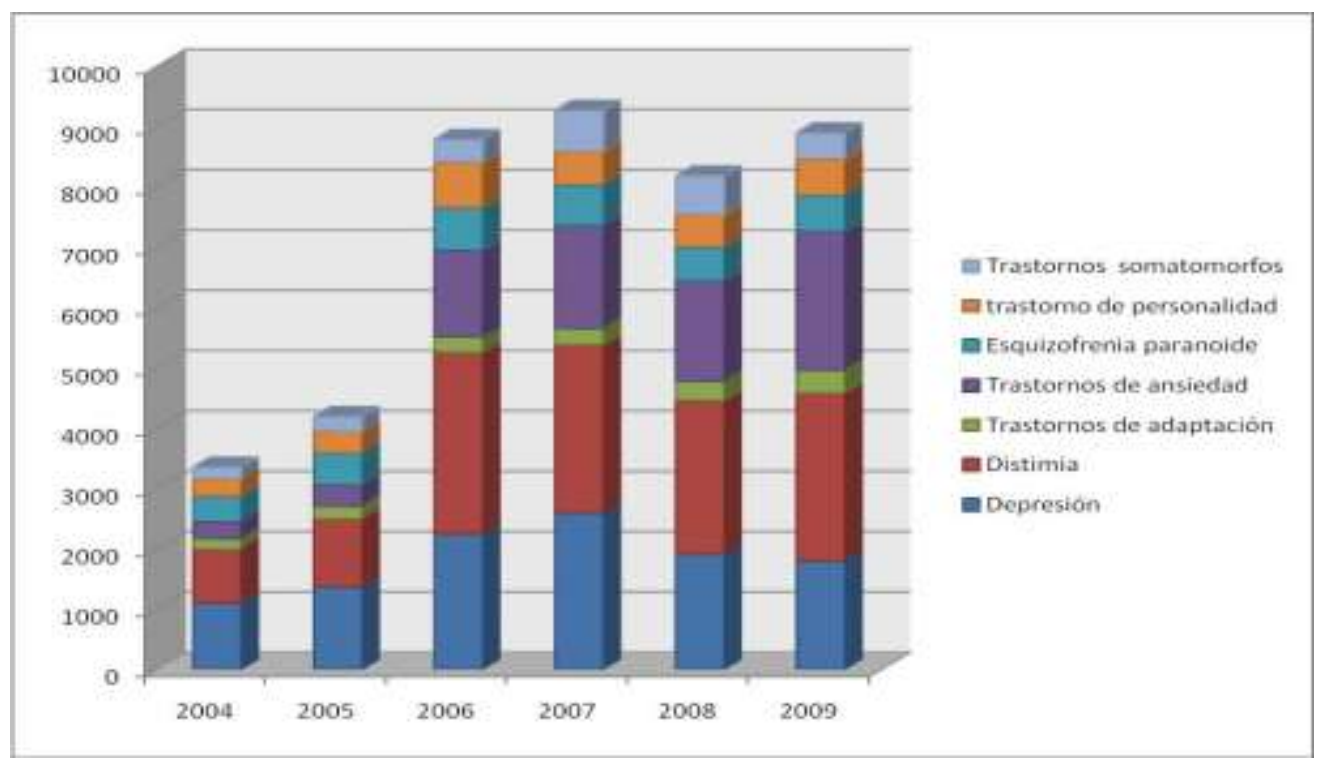

Figura 2. Total de casos diagnosticados de depresión. Fuente: actualidadclinica.wordpress.com con 454,780 pacientes atendidos en el Hospital General de Mexico. 
La gráfica anterior muestra el incremento de los indices de niños con depresion, apartir de las fechas de que la mujer inicia en forma su proceso de desarrollo profesional y con esto vemos que el desarrollo de la mujer, vas a la par con el desarrollo del sindrome de depresion infantil.

Pero en si los indices negativos que se observaron, no se generaron por culpa de la madre o padre de familia, o bien por el hecho de que los mismos se desarrollen profesionalmente, no, el efecto es la falta de atencion y cuidados que se le da al menor, ya que en edades tempranas los niños requieren de una atencion especifica, en (LaHaye, 1979) dice "los niños de los 0 a 8 años están en una etapa de aprendizaje de todo lo que ven y absorven como esponjas, de los 8 a los 12, es su etapa de mayor correccion, formativa y en donde se iniciara el proceso de determinacion de su carácter y de los 12 a los 18, es la etapa en donde concluye su adolescencia y se determina su carácter", por tal motivo, los padres forman una parte escencial en el desarrollo de su hijo. Por tal motivo consideramos que no basta con en hecho de que los padres se desarrollen de manera profesional, sino que a la par, puedan apoyar al desarrollo de los hijos y a su sano crecimiento mental y social.

En (Voltas, 2008) dice que " el educar hoy no es una empresa facil, educar hoy en los valores que una madre- y un padre- consideran como tales para su descendencia, cualquier labor de educacion parece cada vez más ardua y precaria. Por eso se habló de una gran emergencia educativa, de la creciente dificultad para transmitir a las nuevas generaciones los valores fundamentales de la existencia”. Se concordó con este criterio, ya que los niños y/o jovenes de hoy, tienen a su alcance mucha más herramientas y medios para poder llamar a lo malo "bueno" y a lo bueno " malo", pero si los padres en conjunto, como equipo de trabajo, no luchando por la autonomia individual de cada uno, sino por lograr una meta en común, se busca que los valores escenciales vuelvan a florecer en cada familia, se erradicará el síndrome depresivo, o al menos se disminurá al máximo. Los índices de atención médica por concepto de salúd, muestran cifras alarmantes, como lo indican (Rodriguez Avila \& Ortiz Ramirez, 2008) donde dicen "de acuerdo a la OMS en México hay 5,000,000 personas que son tratadas de un problema de salúd pública. De los cuáles del 8 al 10\% son menores de 18 años, señalando que el genero femenino tiene mayor incidencia sobrepasando los 12 años de edad", este es un indicativo muy fuerte, ya que en numeros hablamos de un total de 500,000 niños con problemas de depresión, sólo en nuestro país, indicando que hay por lo tanto en promedio 500,000 hogares con problemas de comunicación, trato y desarrollo social. Aunado a este problema social se puede equiparar la pérdida de valores en general, así como lo menciona (Valdez, 2011):

\section{Causas:}

- La desintegración y los conflictos familiares

- Los divorcios

- La situación económica

- Deserción escolar

- Desobediencia

- Drogadicción

Consecuencias:

- Surgimiento de bandas

- Prostitución

- Embarazos prematuros y no deseados

- Robos

- Transculturación

- Abortos en jóvenes a temprana edad

- Relaciones sexuales promiscuas 
Estos efectos que muestra Valdez, se ven diariamente dentro del esquema de vida, y que encarecen el entorno familiar, social, economico, moral y religioso. Y con todos estos conceptos, solo se pueden ver figuradas las situaciones que día a día que se vive en este mundo, socializado, desarrollado y lleno de afanes, y como se mencionó en paginas anterirores, es sólo uno de tantos transtornos que llevan a perder la visión de la sociedad y la pérdida de valores que se refleja en todas las causas y efectos descritos de manera acertiva por el autor, resumiendo en el esquema en la desintegracion familiar, la perdida de valores y el desinteres por nuestras familias.

\section{RESULTADOS}

No todo esta perdido, contemplando todo lo anterior es tiempo de retomar el camino en el ambito espiritual y moral, doblegando las debilidades y pasiones, y permitiendo que aflore el deseo de mejorar el país, un mejor estado, una mejor ciudad y una mejor familia que es el centro de donde se debe partir. Tal vez la pregunta clave es ¿Cómo? Si los hijos ya son grandes, o tal vez están demasiado pequeños para comprender, o tal vez, en el peor de los casos, ya no están en casa, están recluidos en alguna carcel o simplemente, ya se fueron del hogar a seguir con sus vidas.

Para alcanzar el objetivo de reactivar los valores en la familia se contemplarán algunas estrategias que se pueden seguir, pero primero se definirán qué valores son los que tenemos que seguir para que el proyecto prospere y rinda frutos.

Según (Villarreal Vargas, Seminario Cruz, \& Villarreal Magan, 2011) nos marcan los “ siguientes valores fundamentales :
La alegría: Se siembra primeramente en el seno familiar. Así como marcan los autores, este valor es un "don" con el que no contamos todos, pero que irradia de felicidad el lugar, que se toca, este "don" debe cuidarse como un tesoro, sensible y maravilloso, porque es frágil de romperse y de apagarse, así como el agua apaga el fuego, la alegría puede llegar a desaparecer de nuestra vida.

La generosidad: Actuar a favor de otras personas desinteresadamente y con alegría. Ciertamente situación tan difícil de encontrar como valor hoy en día, ya que siempre se busca el ganar-ganar, "tú me das, yo te doy", si no, no hay pacto, por eso, así como lo marcan los autores, es importante que se desarrolle este valor dentro de la juventud y familiarmente.

El respeto: Se fomentan dentro de la familia, no sólo respeto a la persona misma, sino también a sus opiniones y sentimientos. Valor incalculable en la educación, se ha perdido el respeto a tal grado, de ni siquiera hay respeto propio, por eso, el inculcarlo viene desde los niños, y de los mismos adultos, hacia los niños.

La justicia: Se fomenta en el seno de la familia al establecerse lo que corresponde a cada miembro de la misma. Los autores de manera acertada hablan de la justicia, como valor, y con la importancia que este requiere, ya que esto genera equidad, y la equidad da equilibrio en la vida familiar.

La responsabilidad: Supone asumir las consecuencias de los propios actos, no solo ante uno mismo sino ante los demás. Cuando se es adulto nos motivamos y asumimos la responsabilidad por nuestros actos, de manera inmediata, la inyectamos como valor ante los jóvenes y se logra un pacto de crecimiento mutuo. 
La lealtad: Surge cuando se reconocen y aceptan vínculos que nos unen a otros, de tal manera que se busca fortalecer y salvaguardar dichos vínculos así como los valores que representan. Como mencionan los autores, este valor se representa entre dos grupos, pero nace en el seno de la familia, cuando los padres se respetan y se tiene lealtad uno al otro, la responsabilidad es en común, y representa un torre que difícilmente será quebrantada, la lealtad es uno de los valores que se han ido mermando, en este pasar del tiempo y desarrollo del ser humano.

La autoestima: Uno de los valores fundamentales para el ser humano maduro, equilibrado y sano. Se entiende por autoestima la visión más profunda que cada persona tiene de sí misma, influye de modo decisivo en las elecciones y en la toma de decisiones, en consecuencia conforma el tipo de vida, las actividades y los valores que elegimos.

Valores del espíritu

Fe, oración, vida espiritual, santidad, estado de gracia, apertura a la vocación de los hijos. Este valor importante lo marcan los autores, es una de las partes primordiales de la vida espiritual de los seres humanos, sin fe no podemos crecer y no podemos desarrollarnos.

Los valores del espíritu, nos conducen a llevar una vida sobrenatural, una vida en amistad y comunión con Dios. Nos hacen participantes activos de la iglesia. Situación tal es de suma importancia en la vida, sin los valores espirituales, la vida del ser humano no se puede desarrollar, y menos crecer, es la única forma de desarrollar la formación cívica del ser humano.

Los valores del espíritu dan fruto en la comunidad cristiana haciéndonos apóstoles del Evangelio y participantes de la misión salvadora de Nuestro Señor Jesucristo.
Los valores del espíritu nos ayudan a que la familia sea semillero de vocaciones a la vida sacerdotal y consagrada, y a la santidad en la vida matrimonial.

Valores del dominio de sí mismo

La aceptación y el reconocimiento de este vínculo no se centran hacia el fruto, como una posibilidad, sino que es una realidad actual. Este vínculo no pasa con el tiempo, es profundo, suele madurar y fortalecerse a la larga."

Como se puede observar en el trabajo del Dr. Sadot Villarreal Vargas, expresa de manera clara y precisa la forma en que se representan los valores, y más aún, como podemos hacerlos presentes en las relaciones familiares. Estos valores descritos anteriormente de manera tan correcta, son un antídoto contra los causales de la falta de valores, y buscar que las cosas que están en nuestro alrededor no opaquen nuestro crecimiento y desarrollo.

Conociendo ya los valores más importantes en la sociedad, el segundo paso es buscar emplearlos y adaptarlos a nuestro estilo de vida, buscando una mejor manera de vivir. Tal vez buscando ayuda profesional, como los grupos o asociaciones civiles que se dedican a la promoción de los mismos, tal vez buscando una manera de hacerlos efectivos en el hogar, o buscando un lugar o centro espiritual donde estos sean cultivados y apoyen a los individuos para ponerlos en práctica. En (Rangel Guerrero $\&$ Torres Delgado, 2009) mencionan que "se recomienda para el crecimiento personal en familia:

- Quererse como persona

- Aceptarse como se es

- Saber afrontar con fortaleza las inevitables situaciones negativas de la vida $y$ aprovecharlas para aprender nuevas y más positivas pautas de comportamiento. 
- Tomar distancia de las críticas

- Pensar por sí mismo sobre las diversas situaciones de la vida para poder gozar de criterio propio y personal

- Aceptar y respetar a los demás, porque también ellos son importantes y dignos de ser tenidos en cuenta en sus opiniones y sentimientos, aunque no coincidan con los nuestros

- Reconocer los propios errores con naturalidad, y no castigarse afectivamente por ellos

- Liberar tenciones emocionales y físicas, mediante el dialogo con alguien de confianza, la relajación psicofísica, la distracción, el buen ánimo, el sentido del humor

- Canalizar energías, mediante la creatividad, disfrutar el aire libre y hasta el ejercicio físico

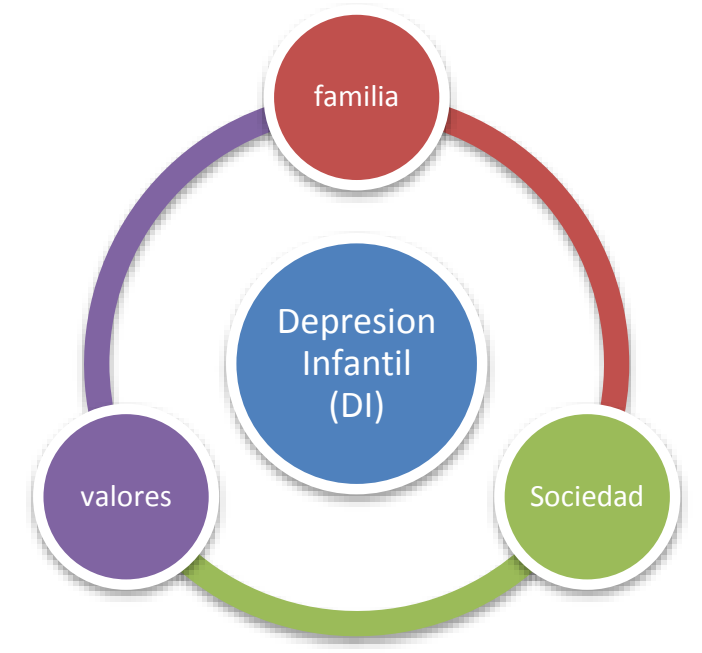

Figura 3. Vinculación entre depresión infantil-familia-valores y sociedad. Fuente: Autoría Propia C.P. y M. A. Marisa Araiza 2012.

Los tres conceptos estan intimamente ligados y representan un eje central en el restablecimiento de la sociedad y del medio de vida. Cuando como seres humanos se logre identificar la importancia que tiene el tiempo de calidad en la vida de las personas, en la atencion a los hijos, no importando las edades que tengan. Se identificará que ellos tiene necesidades, derechos y hambre de ser escuchados, los hijos diario, que se ha demostrado un buen antidepresivo

- Saber disfrutar de lo positivo de la vida, de lo bello, de la amistad, de cantidad de pequeños detalles."

En realidad no es un programa paso a paso para ser feliz, pero si una guía para poder lograr un poco más de estabilidad emocional y afectiva dentro de nuestros hogares, y a la par lograrlo en nuestra comunidad.

\section{CONCLUSIÓN}

Como se puede apreciar, se relacionan tres fases importantes de la vida del ser humano y que cada una de ellas se vincula entre sí: 
a un niño: ¿Qué hará cuándo llega a su casa, disfrutar de una cena rica al lado de sus padres, disfrutar de una plática emosionante con ellos donde pueda contar sus historias de superhéroes o de princesas? $O$ tal vez llegue a un hogar donde los papás cansados del trabajo, sólo quieren ubicarse frente a esa caja de mágia, que embrutece y enmudece su entendimiento llamanda televisión, y el pequeño super- héroe, sólo le quede quitarse la capa, abrir el refrigerador y tomar algo de alimento por su cuenta y la princesa...tal vez se vaya a dormir, en espera de que algun día su principe azul (su padre) tenga un minuto de tiempo para poder recibir un cálido beso en su mejilla.

La documentación sobre los problemas que existen son reales, así que se debe dar un paso atrás y retomar las estrategias que entes se mencionaron para no tener súper héroes frustrados y princesas que se conviertan en brujas. Estamos a tiempo de hacer que el principe azul aparezca y la dama a la que el súper héroe quiere rescatar, está ahí para ser rescatada. Sólo es necesario sencibilizarse a las necesidades de los hijos y hacer lo necesario para reformar los valores failiares, a los hijos y por ende a la sociedad.

No con esto se debe de mermar el desarrollo profesional, sólo hay que adaptar el tiempo, hay que establecer prioridades $y$ sacrificar, tal vez algo, que no da fruto, por algo perecedero....el amor.

Los padres son el pilar de la sociedad, no se puede ser indiferente al alto índice de niños con depresion, a la falta de valores en todos los ambitos de la sociedad y la vida individual. Se debe vivir, sonreir y ser feliz con lo que se tiene, con lo que Dios te da y no reclamar por lo que no se tiene, y disfrutar de las bendiciones que se reciben. Y como dice (Marcos 9:23, 2010) " todo es posible si uno cree..."

\section{LITERATURA CITADA}

Boyd, C. (2003). Hijos diferentes, necesidades diferentes (1a. Ed., Vol. 1). (Unilit, Ed., \& M. Raquel, Trans.) Miami, Florida, USA: Unilit.

HGM. (2011). Actualidad clinica. Mexico: www.actualidadclinica.wordpress.com

Instituto Nacional de Estadísticas: INE. (2007). Enfoque estadistico-mujer y trabajo. Mexico: INE.

LaHaye, B. (1979). Como desarrollar el temperamento de su hijo. Miami, Fl: Unilit.

Marcos 9:23. (2010). la biblia nueva traduccion viviente. miami,flo: tyndale.inc.

Merino, B. P. (2007). Educacion moral en la familia: desafios y oportunidades. REXE: Revista de estudios y experiencias en educacion , 113-124.

Rangel Guerrero, A., \& Torres Delgado, J. M. (2009). la familia funcional como promotora del desarrollo humano integral de la persona desde la perspectiva femenina. (r. e. Scientia, Ed.) Nova Scientia , 2-1 (3), 151-166. https://doi.org/10.21640/ns.v2i3.226

Rodriguez Avila, J. P., \& Ortiz Ramirez, M. D. (2008). Depresion Infantil. (U. A. Hidalgo, Ed.) revista cientifica electronica de Psicologia (6), 77-88.

Valdez, Y. (2011). rincon del vago. Retrieved julio 30, 2012, from http:/html.rincondelvago.com/crisisde-los-valores-tradicionales.html 
Villarreal Vargas, S. D., Seminario Cruz, A. D., \& Villarreal Magan, C. L. (2011). monografias. Retrieved julio 30, 2012, from

http://www.monografias.com/trabajos9

0/relacion-carencia-valores-ycomportamientosinadecuados/relacion-carencia-valores- y-comportamientosinadecuados4.shtml

Voltas, D. (2008). La mujer-madre, un valor que renace. (A. e. medica, Ed.) cuadernos de bioetica , XIX (3), 525532 .

Copyright (c) 2019 Marisa Aideé Araiza Santillán, Mari sol Priscila Palafox Bolivar, Eduar do Dominguez Arrieta, Graciela del Carm en Sandoval Lujan, Octavio Torres López

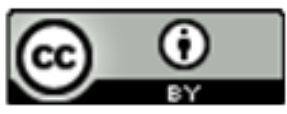

Este texto está protegido por una licencia licencia CreativeCommons 4.0.

Usted es libre para Compartir —copiar y redistribuir el $\mathrm{m}$ aterial en cualquier medio o formato- $\mathrm{y}$ Adaptar el documento —remezclar, transformar y crear a partir del material- para cualquier propósito, incluso para fines com erciales, siempre que cumpla la condición de:

Atribución: Usted debe dar crédito a la obra original de manera adecuada, proporcionar un enlace a la licencia, e indicar si se han realizado cambios. Puede hacerlo en cualquier forma razonable, pero no de forma tal que sugiera que tiene el apoyo del licenciante o 10 recibe por el uso que hace de la obra.

Resumenclelicencia - Textocompletodelalicencia 